\title{
1 Hydrozoan sperm-specific H2B histone variants stabilize 2 chromatin and block transcription without enhancing 3 chromatin condensation
}

4

5 Anna Török ${ }^{1}$, Martin JG Browne ${ }^{1}$, Jordina C Vilar ${ }^{1}$, Indu Patwal ${ }^{1}$, Timothy Q

6 DuBuc $^{1,2}$, Febrimarsa $^{1}$, Erwan Atcheson ${ }^{1}$, Andrew Flaus ${ }^{1}$, Uri Frank ${ }^{1, *}$ and Sebastian

7 G Gornik $^{1,3, *}$

$8{ }^{1}$ Centre for Chromosome Biology, School of Natural Sciences, NUI Galway, Galway, Ireland

$9 \quad{ }^{2}$ Present address: Biology Department, Swarthmore College, Swarthmore, PA 19081, USA

$10{ }^{3}$ Present address: Centre for Organismal Studies, Heidelberg University, 69120 Heidelberg,

11 Germany

12

$13 \quad{ }^{*}$ Correspondence: uri.frank@nuigalway.ie and sebastian.gornik@gmail.com 


\section{Abstract}

16 Many animals achieve sperm chromatin compaction and stabilisation during

17 spermatogenesis by replacing canonical histones with sperm nuclear basic proteins 18 (SNBPs) such as protamines. A number of animals including hydrozoan cnidarians 19 and echinoid sea urchins lack protamines and have instead evolved a distinctive 20 family of sperm-specific histone $\mathrm{H} 2 \mathrm{Bs}$ ( $\mathrm{spH} 2 \mathrm{Bs}$ ) with extended N-termini rich in 21 SPKK-related motifs. Sperm packaging in echinoids such as sea urchins is regulated by $\mathrm{spH} 2 \mathrm{Bs}$ and their sperm is negatively buoyant for fertilization on the sea floor. Hydroid cnidarians also package sperm with $\mathrm{spH} 2 \mathrm{Bs}$ but undertake broadcast spawning and their sperm properties are poorly characterised. We show that sperm chromatin from the hydroid Hydractinia possesses higher stability than its somatic equivalent, with reduced accessibility of sperm chromatin to transposase $\operatorname{Tn} 5$ integration in vivo and to endonucleases in vitro. However, nuclear dimensions are only moderately reduced in mature Hydractinia sperm compared to other cell types.

29 Ectopic expression of $\mathrm{spH} 2 \mathrm{~B}$ in the background of $\mathrm{H} 2 \mathrm{~B}$ knockdown resulted in 30 downregulation of global transcription and cell cycle arrest in embryos without 31 altering their nuclear density. Taken together, spH2B variants containing SPKKrelated motifs act to stabilise chromatin and silence transcription in Hydractinia sperm without significant chromatin compaction. This is consistent with a contribution of $\mathrm{spH} 2 \mathrm{~B}$ to sperm buoyancy as a reproductive adaptation.

\section{Keywords}

36 Histone variants, chromatin, H2B, sperm-specific histones, SPKK motif, T/SPXZ motif, SPXK motif. 


\section{Introduction}

Male haploid sperm are produced from diploid progenitors. In this process, progenitor chromatin undergoes major remodelling to produce mature sperm chromatin exhibiting a high degree of condensation and stability compared with somatic chromatin (Sassone-Corsi, 2002; Ward and Coffey, 1991). This is achieved, in part, by sperm nuclear basic proteins (SNBPs) that replace somatic histones. SNBPs are structurally heterogeneous across species and are classified as histone-related, protamines, or protamine-related proteins (Török and Gornik, 2018).

Sequenced hydrozoan cnidarian genomes lack genes for protamines and instead encode a number of sperm-specific histone $\mathrm{H} 2 \mathrm{~B}$ variants ( $\mathrm{spH} 2 \mathrm{Bs}$ ) that are structurally related to a protein family previously characterised in echinoid sea urchins (Ausio, 1999; Ausio et al., 1997; Busslinger and Barberis, 1985; Marzluff et al., 2006; Pérez-Montero et al., 2016; Poccia and Green, 1992; Rocchini et al., 1996; Török et al., 2016) (Fig. 1A). These histones include an extended N-terminal tail with up to 7 repeats of a $\mathrm{SP}[\mathrm{K} / \mathrm{R}][\mathrm{K} / \mathrm{R}]$ motif known to bind with high affinity to DNA minor grooves, preferentially at A/T rich sequences (Churchill and Suzuki, 1989; Khadake and Rao, 1997; Suzuki, 1989; Suzuki et al., 1993; Suzuki et al., 1990). Phosphorylation of the serine on the SPKK-related motifs in sea urchins inhibits DNA-binding (Green and Poccia, 1985; Poccia and Green, 1992). SPKK-related motif containing spH2Bs change the condensation status of sperm chromatin in echinoids and make chromatin inaccessible for transcription (Poccia and Green, 1992; Poccia et al., 1987).

We have previously shown that $\mathrm{spH} 2 \mathrm{Bs}$ are expressed in male gonads of the hydrozoan cnidarian Hydractinia echinata (Török et al., 2016). Here, we characterise the Hydractinia spH2Bs biochemically and functionally using the sibling species $H$. symbiolongicarpus. We complement this with in vitro observations of the properties of mononucleosomes and oligonucleosomal arrays containing recombinant Hydractinia spH2Bs. Our results show that $\mathrm{spH} 2 \mathrm{Bs}$ inhibit transcription and stabilize chromatin without contributing significantly to its compaction. These characteristics are consistent with a function to facilitate fertilization in these broadcast-spawning animals. 


\section{$71 \quad$ Results}

\section{spH2Bs are co-expressed and replace H2B.1 during spermatogenesis} but do not significantly increase chromatin compaction

Hydractinia echinata and H. symbiolongicarpus both possess several hundred, tandemly repeated copies of a single canonical H2B-encoding gene (H2B.1) but only four non-canonical sperm-specific H2B variant (H2B.3, H2B.4, H2B.5, H2B.6; Fig. 1B); the latter containing 5-7 N-terminal SP[K/R][K/R] repeats (Török et al., 2016). The H2B.3 and H2B.4 pair, and the H2B.5 and H2B.6 pair, are each identical in protein sequence but differ slightly at the nucleotide level and are encoded at unique chromosomal loci (Török et al., 2016). We refer to all 4 variants collectively as $\mathrm{spH} 2 \mathrm{Bs}$, except where a specific member is investigated. For in vitro experiments, we investigated the properties of $H$. echinata H2B.3 and H2B.6 variants as representatives of Hydractinia $\mathrm{spH} 2 \mathrm{~B}$ pairs and compared them with canonical H2B.1.

We used fluorescence in situ hybridization (FISH) to confirm that the expression patterns of $\mathrm{spH} 2 \mathrm{Bs}$ in $H$. symbiolongicarpus were equivalent to the previously published $H$. echinata expression. As reported (Török et al., 2016), the high nucleotide sequence similarity between $H 2 B .3$ and $H 2 B .4$, and between $H 2 B .5$ and $H 2 B .6$, precludes the design of gene-specific cRNA probes so we treated each pair as a combined case. We observed that $H 2 B .3 / 4$ and $H 2 B .5 / 6$ were co-expressed in sperm progenitor cells within male gonads of $H$. symbiolongicarpus (Fig. 2A) while the expression of H2B.1 was concomitantly downregulated (Török et al., 2016), consistent with its replacement by $\mathrm{spH} 2 \mathrm{Bs}$ during spermatogenesis.

To test whether $\mathrm{spH} 2 \mathrm{Bs}$ affect overall genome compaction relative to $\mathrm{H} 2 \mathrm{~B} .1$, we measured the nuclear dimensions of sperm cells relative to somatic cells in $H$. symbiolongicarpus. Nuclei of male sexual polyps were stained with Hoechst and confocal images were taken of somatic cells and of cells in the late stage of spermatogenesis (Fig 2B). Then, the cross-section areas of multiple individual nuclei were calculated. Nuclei of diploid somatic cells had a median area that was $50 \%$ larger than haploid mature sperm nuclei (Fig 2C). This is consistent with little or no volume reduction of chromatin involving spH2Bs comparing with 6-8-fold volume reduction in protamine-compacted sperm chromatin (Fuentes-Mascorro et al., 2000). 
103 To further investigate the DNA compaction by spH2Bs, we assembled nucleosomal 104 arrays from Hydractinia histones. Oligonucleosome arrays containing 12 repeats of 105 the well-characterised Widom 601 strong nucleosome positioning sequence (Dorigo 106 et al., 2003; Lowary and Widom, 1998) were assembled with recombinant 107 Hydractinia histone octamers containing either H2B.1, H2B.3, or H2B.6. Native 108 PAGE analysis of these nucleosomal arrays did not show any significant difference in 109 the electrophoretic mobility (Fig. 2D). To ensure that this was not an artifact of the 110177 bp Widom 601 sequence repeat, we also prepared equivalent oligonucleosome 111 arrays on the $12 \times 208$ bp repeat Simpson 5S sequence (Simpson et al., 1985) and on a 112 native non-repetitive 2080 bp Hydractinia genomic AT-rich DNA sequence with 113 equivalent results (Fig. 2E). We conclude that $\mathrm{spH} 2 \mathrm{Bs}$ do not lead to significantly 114 enhanced chromatin compaction compared to H2B.1 in vitro or in vivo.

$116 \mathrm{SP}[\mathrm{K} / \mathrm{R}][\mathrm{K} / \mathrm{R}]$ motifs stabilise chromatin structure and restrict 117 chromatin accessibility

118 To observe the effect of $\mathrm{spH} 2 \mathrm{Bs}$ on nucleosome stability, we assembled 119 mononucleosomes using recombinant Hydractinia histone octamers containing 120 H2B.1, H2B.3, or H2B.6 separately as mononucleosomes and measured their thermal 121 stability (Taguchi et al., 2014). DNA was fully released from all mononucleosomes at $12288-90^{\circ} \mathrm{C}$, and nucleosomes containing canonical H2B.1 showed a maximum of $\mathrm{H} 2 \mathrm{~A}-$ $123 \mathrm{H} 2 \mathrm{~B}$ dimer dissociation at $70^{\circ} \mathrm{C}$ (Fig. 3A), consistent with other metazoan canonical 124 H2B-containing nucleosomes (Taguchi et al., 2014). In contrast, nucleosomes 125 incorporating either H2B.3 or H2B.6 showed more progressive thermal dissociation 126 of $\mathrm{H} 2 \mathrm{~A}-\mathrm{H} 2 \mathrm{~B}$ dimers, reaching a maximum at $78^{\circ} \mathrm{C}$ (Fig. 3A).

127 To probe the impact of $\mathrm{spH} 2 \mathrm{Bs}$ on chromatin accessibility in vivo, we performed 128 nuclease accessibility assays and observed an increase in nucleosomal repeat lengths 129 after micrococcal nuclease (MNase) digestion of mature sperm cells compared to 130 progenitors, consistent with a reduced accessibility of linker DNA between spH2B131 containing nucleosomes (Green and Poccia, 1988; Hill and Thomas, 1990) (Fig. 3B). 132 We then performed ATAC-see on these nuclei and observed a strong signal from 133 sperm progenitors whereas DNA was inaccessible to Tn5 transposase in mature sperm 134 (Fig. 3C). This demonstrates that sperm chromatin has reduced accessibility in vivo. 
135 Finally, we performed parallel in vitro ApoI restriction enzyme digestion on

136 oligonucleosomal arrays assembled in vitro on a native $2080 \mathrm{bp}$ non-repetitive 137 Hydractinia DNA fragment with H2B.1, H2B.3, or H2B.6 containing histone 138 octamers. ApoI digestion at 8 recognition sites along the DNA was quantitatively 139 slower for H2B.3 or H2B.6-containing arrays than for H2B.1-containing arrays (Fig. $1403 \mathrm{D}$ and $3 \mathrm{E}$ ), in agreement with the reduction of accessibility seen in vivo.

spH2Bs cause a transcription block and cell cycle arrest in the absence

144 To test the functional effects of $\mathrm{spH} 2 \mathrm{~B}$ incorporation in chromatin we injected in vitro 145 transcribed mRNA encoding the full-length sequences of $H 2 B .3, H 2 B .4, H 2 B .5$, and 146 H2B.6 fused to GFP together into one blastomere of two-cell stage embryos and 147 compared this to injections of mRNA encoding GFP alone as a control. All embryos 148 showed GFP fluorescence 6 hours after injection in 50\% of cells (Fig. 4A). Animals 149 injected with the combined spH2B-GFP fusion mRNAs exhibited nuclear 150 fluorescence whereas control GFP alone was cytoplasmic (Fig. S1). Embryos 151 developed into planula larvae within 2-3 days, similar to untreated Hydractinia 152 embryos.

153 We hypothesized that the injected mRNAs encoding spH2Bs would be outnumbered 154 by endogenous $H 2 B .1$ transcripts because there are some 700 copies of the canonical $155 \quad H 2 B .1$ gene in the Hydractinia haploid genome but only single copies of each spH2B 156 variant (Török et al., 2016). Therefore, we co-injected an H2B.1-specific translation 157 blocking morpholino together with the mRNAs for the four spH2B variants (Fig. S2). 158 Control morpholinos had no effect on the embryos (Fig. 4B) and injecting the 159 morpholino alone led to cell cycle arrest and death of all embryos within a few cell 160 cycles (Fig. 4C). This could be partially rescued by co-injection of morpholino161 resistant H2B.1 encoding mRNA (Fig. 4D and Fig. S2), confirming the specificity of 162 the morpholino.

163 We then injected $H 2 B .3, H 2 B .4, H 2 B .5$, and $H 2 B .6$ mRNAs individually along with 164 the H2B.1-specific morpholino. This caused cell cycle arrest equivalent to the 165 injection of the morpholino alone, showing that spH2Bs cannot substitute for H2B.1 
166 (Fig. 4E-I). Finally, we used ethynyl uridine (EU) incorporation to quantitate nascent

167 RNA levels and found them to be significantly lower in H2B.6 mRNA-injected 168 embryos than in embryos co-injected with the $H 2 B .1$ rescue mRNA in morphants 169 (Fig. 4J-N).

170 Therefore, the mRNA injection experiments are consistent with $\mathrm{spH} 2 \mathrm{~B}$ incorporation 171 in chromatin in place of H2B.1 leading to a generalised transcriptional block.

\section{Discussion}

174 Sperm-specific histones H2B.3, H2B.4, H2B.5 and H2B.6 containing N-terminal $175 \mathrm{SP}[\mathrm{K} / \mathrm{R}][\mathrm{K} / \mathrm{R}]$ repeats increased chromatin stability and reduced DNA accessibility 176 compared with chromatin assembled with canonical H2B.1 both in vivo and in vitro.

177 Our observations are consistent with transcriptional silencing resulting from networks 178 of linker DNA binding of $\mathrm{spH} 2 \mathrm{Bs}$ in sperm, in line with earlier investigations with 179 structurally similar H2B variants in sea urchin sperm. However, given the suggested 180 ease of evolving SPKK-related motifs (Malik et al., 2002) and the fact that spH2Bs 181 are only known in two unrelated taxa, we suggest that hydrozoan and echinoid 182 spH2Bs evolved convergently.

183 Protamine-type SNBPs generate highly compact nuclei and this has been suggested to 184 improved sperm motility (Champroux et al., 2016; Levitan and Petersen, 1995). In 185 contrast, Hydractinia $\mathrm{spH} 2 \mathrm{Bs}$ do not appear to increase chromatin density. 186 Hydractinia colonies are dioecious, growing individually on the surface of hermit 187 crab shells. Their spawning is light induced but the distance to a potential mate 188 depends on the behaviour of their host hermit crabs. Eggs are near neutrally buoyant 189 upon spawning, sinking only very slowly to the bottom in calm water (Video S1) and 190 remaining suspended under turbulent conditions. This feature probably prevents egg 191 loss in the sediment, but fertilization would be highly unlikely unless sperm have comparable buoyancy. Upon fertilization, zygotes become strongly negatively 193 buoyant (Video S2; Fig. S3), probably to protect the embryo from predation in the 194 water column and allow the larvae to attach to benthic hermit crab shells. Since 195 chromatin contributes a major part of total sperm biomolecule composition, we 196 speculate that by maintaining reduced accessibility without increasing compaction, 


\section{Material and Methods} evolution of hydrozoan spH2Bs.

\section{Animal culture} harvested from mature colonies.

\section{Microinjection} completed before the first cell division.

\section{Genomic DNA extractions}

As previously described (Török et al., 2016).

\section{Micrococcal nuclease (MNase) assay}

As previously described (Török et al., 2016).

\section{Fluorescent in situ hybridisation (FISH)}

spH2Bs could contribute to neutral Hydractinia sperm buoyancy. Less dense sperm would increase fertilization likelihood and could be a selective pressure that drove the

Hydractinia colonies were cultured in artificial seawater at $18^{\circ} \mathrm{C}$ under $14 \mathrm{~h}: 10 \mathrm{~h}$ lightdark regimes and fed Artemia franciscana nauplii four times a week, and ground oyster once per week. Spawning is light induced, occurring synchronously in both sexes (Frank et al., 2020). Fertilised eggs were collected for microinjection and developed at room temperature into planula larvae within 3 days. Polyps were

Injection needles were prepared from glass capillaries (Narishige GD-1 1x90 mm) using a microneedle puller (Narishige; cat no.: PN-31) with settings of heat 560, pull 70, velocity 75 , time 150 . Microinjection was carried out immediately after fertilisation in 1-cell stage embryos. Embryos were placed in a small Petri dish lid with a $200 \mu \mathrm{m}$ plankton net attached to avoid any movement. Injections were

As previously described (Török et al., 2016). 


\section{Capped and polyadenylated mRNA synthesis}

For mRNA synthesis the desired fragment including $100 \mathrm{bp} 5$ '-UTR and the coding sequence of the histone gene was amplified by PCR from genomic DNA. Linker sequences and GFP tags were added to the construct by Gibson assembly. The assembled fragment was re-amplified by PCR, with primers containing T7 promoter necessary for RNA synthesis. HiScribe T7 ARCA mRNA kit (NEB) was used for mRNA synthesis with $10 \mu \mathrm{l}$ of $2 \mathrm{x}$ ARCA/NTP mix, $1 \mu \mathrm{g}$ of template DNA, $2 \mu \mathrm{l}$ of T7 RNA Polymerase mix and nuclease-free water to a final volume of $20 \mu \mathrm{l}$. Reactions were gently mixed and incubated at $37^{\circ} \mathrm{C}$ for $1-2$ hours. $2 \mu \mathrm{l}$ DNaseI was added to the mixtures and incubated for a further 15 min. PolyA tailing was carried out in a total volume of $100 \mu \mathrm{l}$ with $20 \mu \mathrm{l}$ of IVT reaction (from above), $65 \mu$ l of nuclease-free water, $10 \mu \mathrm{l}$ of $10 \mathrm{x}$ Poly(A) Polymerase Reaction Buffer and $5 \mu$ of Poly(A) polymerase. Reactions were mixed and incubated at $37^{\circ} \mathrm{C}$ for $30 \mathrm{~min}$. In order to purify mRNA 0.5 volume of $\mathrm{LiCl}$ solution (7.5 M LiCl, $10 \mathrm{mM}$ EDTA) was added to each sample and incubated at $-20^{\circ} \mathrm{C}$ for $30 \mathrm{~min}$. Tubes were centrifuged at $4^{\circ} \mathrm{C}$ for 15 min at full speed. Supernatant was removed and pellets were rinsed with $500 \mu$ cold ethanol. Tubes were centrifuged again at $4^{\circ} \mathrm{C}$ for $10 \mathrm{~min}$ at full speed. Ethanol and residual liquid was carefully removed using a sharp tip then heated to $65^{\circ} \mathrm{C}$ for $5-10$ min to completely dissolve RNA. Following this, mRNA concentrations were measured and the tubes were stored at $-80^{\circ} \mathrm{C}$. mRNA could be visualised and analysed on formaldehyde denaturing gels.

\section{EdU staining of S-phase cells}

EdU visualisation was performed using a Click-iT EdU Alexa Fluor 488 Imaging kit (ThermoFisher Scientific). The solutions were prepared according to the manufacturer's instructions. Fluorescence excitation and emission maxima for Alexa Fluor 488 were 495 and $519 \mathrm{~nm}$ respectively. For EdU staining animals were incubated in EdU solution for $30 \mathrm{~min}$ at a concentration of $150 \mu \mathrm{M}$. Following incubation FISH was performed as described above when it was necessary. Animals were then fixed in 4\% PFA in $100 \mathrm{mM}$ HEPES pH 7.5, $4 \mathrm{mM} \mathrm{MgSO} 4,140 \mathrm{mM} \mathrm{NaCl}$ overnight at $4^{\circ} \mathrm{C}$. Following this, animals were washed twice in $3 \%$ BSA/PBSTx $(0.1 \%)$ for 15 min each. The sample was permeabilised by incubation in $0.5 \%$ PBSTx for 1 hour. Animals were then washed twice for $10 \mathrm{~min}$ in 3\% BSA/PBSTx $(0.1 \%)$. 
254 Staining cocktail containing $430 \mu \mathrm{l}$ of $1 \mathrm{x}$ reaction buffer, $20 \mu \mathrm{l} \mathrm{CuSO}_{4}, 1.2 \mu \mathrm{l}$ Alexa

255 Fluor 488 azide and $50 \mu \mathrm{l}$ of reaction buffer additive in a total volume of $0.5 \mathrm{ml}$ was

256 prepared. The reaction cocktail was then added to the animals to cover them entirely

257 and incubated in the dark for $30 \mathrm{~min}$ at RT then washed four times in 3\% BSA/PBS

258 Triton $(0.1 \%)$ for $15 \mathrm{~min}$ each. Nuclei were stained in $10 \mathrm{ng} \mu^{-1}$ Hoechst 33258

259 (Sigma B2883) in PBSTx for 15 min followed by one wash in PBSTx for 10 min.

260 Samples were mounted on microscopic glass slides using Fluoroshield mounting 261 medium (Sigma F4680), sealed with nail polish and stored at $-20^{\circ} \mathrm{C}$. Images were 262 taken on Olympus FV1000 inverted confocal microscope.

\section{EU staining to detect nascent RNA}

264 EU visualisation was performed using a Click-iT RNA Imaging Kit (ThermoFisher

265 Scientific). The solutions were prepared according to the manufacturer's instructions.

266 For EU staining embryos were incubated in EU solution for 1 hour at a final 267 concentration of $0.5 \mathrm{mM}$ and fixed in 4\% PFA in HEPES for 1 hour at RT. Embryos 268 were then washed in PBS for $5 \mathrm{~min}$ followed by quick washes in increasing 269 concentrations of methanol. Animals were first placed in $25 \%$ methanol $\mathrm{v} / \mathrm{v}$ in PBS 270 followed sequentially by $50 \%, 75 \%$ and $100 \%$ methanol. The embryos were then 271 rehydrated by washing in decreasing concentrations of $\mathrm{MeOH}$. First in $75 \% \mathrm{MeOH}$, 272 then in 50\% methanol and finally in $25 \%$ methanol for a few seconds each. Embryos 273 were washed once again in PBS for 5 min. To permeabilise the samples, the embryos 274 were incubated in $0.5 \%$ TritonX-100 in PBS for 15 min at RT. The permeabilisation 275 buffer was removed and the embryos were washed once with PBS for 5 min. Staining cocktail was prepared to a total volume of $250 \mu$ containing $214 \mu \mathrm{l}$ of $1 \mathrm{x}$ Click-iT RNA reaction buffer, $10 \mu \mathrm{CuSO} 4,1 \mu \mathrm{l}$ Alexa Fluor 488 azide and $25 \mu$ Click-iT reaction buffer additive. The reaction cocktail was added to the embryos to cover 279 them entirely. The animals were incubated for $30 \mathrm{~min}$ at RT, protected from light. 280 Following incubation animals were washed four times in PBS for 15 min each. Nuclei 281 were stained in $10 \mathrm{mg} \mathrm{ml}^{-1}$ DAPI in PBS for $15 \mathrm{~min}$. Animals were washed in $0.3 \%$ 282 TritonX-100 in PBS for 10 min. Samples were then mounted on microscopic glass 283 slides using Fluoroshield mounting medium (Sigma F4680), sealed with nail polish 284 and stored at $-20^{\circ} \mathrm{C}$. Images were taken on Olympus FV1000 inverted confocal 285 microscope. 


\section{Microscopy}

An Olympus SZX7 stereomicroscope was used for animal observation, colony maintenance and injection. Fluorescence microscopy was carried out using an Olympus BX51 compound microscope. Images were processed with the Olympus CellD software package. Confocal microscopy was carried out on the Olympus Fluoview 1000 software with an inverted IX71 microscope. In order to reduce noise the Kalman accumulation function was applied. Images were then acquired and saved. In order to observe the desired multiple sections the Z-position was also adjusted. The stacks of sections could then be projected together. Image analysis was carried out using ImageJ Software.

\section{Morpholino mediated knockdown}

Morpholino oligos were designed around the start codon of the gene of interest using Gene Tools software (http://www.gene-tools.com) (Fig. S1A). Morpholino oligos were diluted to the recommended concentrations and injected into 1-cell stage embryos. Control morpholino sequence: CCTCTTACCTCAgTTACAATTTATA. H2B.1 morpholino sequence: GCTGCTGCGTCAGACATGGTTAAAT.

\section{Assay of transposase-accessible chromatin with visualisation (ATAC-see)}

Hyperactive Tn5 transposase was produced and purified as described (Picelli et al., 2014). High-quality, tagmentation-ready Tn5 transposase was then loaded with DNA adaptors that selectively bind to accessible chromatin only (Buenrostro et al., 2013). Tn5 transposome assembly was carried out by resuspending Tn5ME-AATTO590, Tn5ME-B-ATTO590 and Tn5MErev oligos in nuclease free water to a final concentration of $100 \mu \mathrm{M}$. Equimolar amounts of Tn5MErev/Tn5ME-A-ATTO590 and Tn5MErev/Tn5ME-B-ATTO590 were mixed in separate $200 \mu \mathrm{l}$ PCR tubes and incubated at $95^{\circ} \mathrm{C}$ for 5 min then cooled down slowly by turning off the thermocycler. The assembly of transposase solution was carried out in dark using 0.25 volumes of Tn5MErev/Tn5ME-A-ATTO590 + Tn5MErev/Tn5ME-B-ATTO590 at $50 \mu \mathrm{M}$ final concentration, 0.4 volume of $100 \%$ sterile glycerol, 0.12 volume of sterile filtered $2 \mathrm{x}$ dialysis buffer (100 mM HEPES-KOH at pH 7.2, 0.2 M NaCl, 0.2 mM EDTA, $2 \mathrm{mM}$ DTT, $0.2 \%$ Triton X100, 20\% glycerol), 0.1 volume of $50 \mu \mathrm{M}$ SL-Tn5 and 0.13 volumes of water. Reagents were gently mixed, and incubated at RT for $1 \mathrm{~h}$, allowing 
the oligos to anneal to Tn5. Transposase solution was stored at $-20^{\circ} \mathrm{C}$. Slide preparation and fixation was carried outby fixing Hydractinia sperm cells and pronase-treated somatic cells with $1 \%$ formaldehyde for $10 \mathrm{~min}$ at RT. Sperm cells were fixed on glass coverslips using Cytospin, however this could not be performed with somatic cells as it damaged their structure. ATAC-see staining was carried out by premeabilising with lysis buffer $(10 \mathrm{mM}$ Tris-Cl, $\mathrm{pH} 7.4,10 \mathrm{mM} \mathrm{NaCl}, 3 \mathrm{mM}$ $\mathrm{MgCl} 2,0.01 \%$ Igepal CA-630) for $10 \mathrm{~min}$ at RT. The samples were rinsed with $1 \mathrm{x}$ PBS twice and placed in a humid chamber box at $37^{\circ} \mathrm{C}$. The transposase mixture solution $(25 \mu 12 \mathrm{x}$ TD buffer, final concentration of $100 \mathrm{nM}$ Tn5ATTO-59ON in a volume of $50 \mu \mathrm{l}$ ) was placed on the cells and incubated for $30 \mathrm{~min}$ at $37^{\circ} \mathrm{C}$. The slides were washed with $1 \times$ PBS containing $0.01 \%$ SDS and $50 \mathrm{mM}$ EDTA for 15 min three times at $55^{\circ} \mathrm{C}$. Nuclei were stained in $10 \mathrm{ng} \mu 1^{-1}$ Hoechst 33258 (Sigma B2883) then mounted using Fluoroshield medium (Sigma F4680) for confocal imaging.

\section{Recombinant histone expression}

All histone genes except $\mathrm{H} 4$ were amplified from Hydractinia genomic DNA using degenerate primers and subcloned into pET3a. The H4 coding sequence was optimized and synthesized in pD451 (Suppl. File S1). E. coli Rosetta2 pLysS or Star pRIL cells chosen for optimal expression were transformed with expression plasmids and plated on LB agar containing appropriate antibiotics then grown overnight at $37^{\circ} \mathrm{C}$. Colonies were transferred to $1 \mathrm{~L}$ of $2 \mathrm{YT}$ media supplemented with antibiotics and grown until OD600 0.6-0.8. Expression was induced by the addition of IPTG to a final concentration of $0.4 \mathrm{mM}$. Cultures were grown for a further $4 \mathrm{~h}$ at $37^{\circ} \mathrm{C}$ and 180 rpm. Cells were then harvested by centrifugation at 5,000 $\mathrm{G}$ for $15 \mathrm{~min}$ and cell pellets were stored at $-20^{\circ} \mathrm{C}$.

\section{Recombinant histone purification}

The cell pellet containing histone inclusion bodies were allowed to thaw and resuspended in $30 \mathrm{ml}$ Histone Wash Buffer (50 mM Tris-HCl pH7.5, $100 \mathrm{mM} \mathrm{NaCl}$, $5 \mathrm{mM} \beta$-mercaptoethanol, $1 \mathrm{mM}$ benzamidine hydrochloride). Cell suspensions were sonicated in a Branson Sonifier 250 with $12 \mathrm{~mm}$ tip on ice for $1 \mathrm{~min}$ at $40 \%$ amplitude with $5 \mathrm{~s}$ on pulses and $10 \mathrm{~s}$ off pulses, and the lysate was centrifuged at $30,000 \mathrm{G}$ for $15 \mathrm{~min}$. The resulting pellet was washed several times with Histone Wash Buffer, resuspended in $0.5 \mathrm{ml}$ DMSO and incubated at RT for $30 \mathrm{~min}$. The 
inclusion bodies were further solubilised by the addition of $5 \mathrm{ml}$ of Unfolding Buffer (20 mM Tris- $\mathrm{HCl} \mathrm{pH}$ 7.5, 7 M Guanidine HCl, 10 mM DTT) and incubated on rollers for $1 \mathrm{~h}$ at RT. Following this, Histone Purification Buffer A (50 mM Tris-HCl pH 7.5, $7 \mathrm{M}$ Urea, $1 \mathrm{mM}$ EDTA) was added to a final volume of $20 \mathrm{ml}$ and centrifuged at $35,000 \mathrm{G}$ for $20 \mathrm{~min}$. The resulting supernatant was centrifuged for $20 \mathrm{~min}$ at 35,000 G. This final supernatant was then added to $55 \mathrm{ml}$ of Histone Purification Buffer A. The histone solution was passed through a PVDF filter with $0.2 \mu \mathrm{m}$ pore size attached to a vacuum bottle. Samples were then loaded onto a $5 \mathrm{ml}$ SP Sepharose HP column using an FPLC system. Histones were eluted with a linear gradient of increasing Histone Purification Buffer B (50 mM Tris-HCl pH 7.5, 7 M Urea, 1 mM EDTA, 2 M $\mathrm{NaCl})$.

\section{SDS polyacrylamide gel electrophoresis (SDS-PAGE)}

SDS polyacrylamide gels were made to a final acrylamide concentration of $15 \%$ acrylamide with 1:37.5 bis-acrylamide. Samples were resuspended in the presence of 1x protein gel loading buffer and heated to $95^{\circ} \mathrm{C}$ for 5 min prior to loading. Gel electrophoresis was carried out by applying a constant $100 \mathrm{~V}$ in $1 \times$ TG buffer $\left(30 \mathrm{~g} \mathrm{~L}^{-}\right.$ ${ }^{1}$ Tris, $144 \mathrm{~g} \mathrm{~L}^{-1}$ glycine). Protein bands were visualised by Coomassie blue staining.

\section{Histone octamer refolding by salt dialysis}

Equal quantities of lyophilised histones were resuspended in Unfolding Buffer to a final concentration of $2 \mathrm{mg} \mathrm{ml}^{-1}$. Denaturing resuspension was carried out at RT on a roller for $1 \mathrm{~h}$ then centrifugation at 18,000 $\mathrm{G}$ for $10 \mathrm{~min}$. The concentration of each histone supernatant was determined using calculated extinction coefficients (Expasy ProtParam). The four histones were mixed in equimolar ratios and the volume adjusted to a final protein concentration of $1 \mathrm{mg} \mathrm{ml}^{-1}$. This mixture was placed in an 8,000 MWCO dialysis bag and twice dialysed against $600 \mathrm{ml}$ of Refolding Buffer (10 $\mathrm{mM}$ Tris- $\mathrm{HCl} \mathrm{pH} 7.5,2 \mathrm{M} \mathrm{NaCl}, 1 \mathrm{mM}$ EDTA, $5 \mathrm{mM} \beta$-mercaptoethanol) at $4{ }^{\circ} \mathrm{C}$ for $3 \mathrm{~h}$ each followed by a third overnight dialysis. After concentration to $1 \mathrm{ml}$ using a Millipore Ultrafree 10K MWCO concentrator, histone octamers were purified by size exclusion chromatography using a Superdex 200 column equilibrated in Refolding Buffer. Fractions containing all 4 histones at equimolar ratios were confirmed by SDS PAGE and pooled then concentrated to approx. $10 \mu \mathrm{M}$ using a Millipore Ultrafree 10K MWCO concentrator. 


\section{DNA preparation by plasmid digestion}

Plasmids containing 12x 177 bp 601 (Dorigo et al., 2003) and pCL3 containing 11x 208 bp 5S rDNA DNA repeats (Logie and Peterson, 1997) were transformed into $E$. coli TOP10 cells then grown in large scale LB media cultures with appropriate antibiotics. Plasmids were extracted from harvested cells by using ThermoFisher GeneJet plasmid DNA purification kits.

The 601 repeat insert was isolated by digesting purified plasmid at $37^{\circ} \mathrm{C}$ overnight using $0.34 \mathrm{NEB}$ units $\mu \mathrm{g}^{-1}$ of EcoRV. The $5 \mathrm{~S}$ repeat insert was isolated by digesting pCL3 with EcoRV, BgII and BstXI at $37^{\circ} \mathrm{C}$ overnight using $0.34 \mathrm{NEB}$ units $\mu \mathrm{g}^{-1}$ of each enzyme. The $2.1 \mathrm{kbp}$ and $2.3 \mathrm{kbp}$ inserts were isolated from smaller plasmid fragments after electrophoresis in $0.7 \%$ agarose gels run in 1x TAE buffer (40 mM Tris-acetate $\mathrm{pH}$ 8.0, 1 mM EDTA) using a ThermoFisher GeneJet gel extraction kit.

Finally, DNA fragments were loaded onto a $5 \mathrm{~mL}$ MonoQ ion exchange column and eluted by a linear gradient of DNA binding buffer B (20 mM Tris-HCl pH7.5, 0.1 mM EDTA, $2 \mathrm{M} \mathrm{NaCl}$ ) over 5 column volumes. The desired peak fractions were identified by $1 \%$ agarose gel electrophoresis, then ethanol precipitated and the pellet was resuspended in $10 \mathrm{mM}$ Tris- $\mathrm{HCl} \mathrm{pH}$ 7.5.

\section{DNA preparation by PCR}

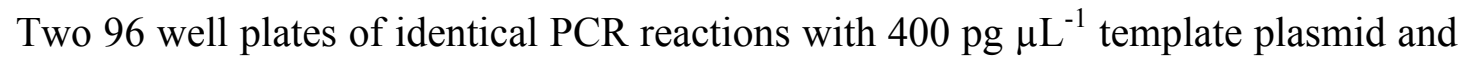
$400 \mu \mathrm{M}$ dNTPs in Taq buffer (10 mM Tris- $\mathrm{HCl} \mathrm{pH} 9.0,50 \mathrm{mM} \mathrm{KCl}, 1.5 \mathrm{mM} \mathrm{MgCl}$, $0.1 \%$ Triton X-100) with were amplified using Taq polymerase with $800 \mathrm{nM}$ primers. Template plasmids containing a 2080 bp non-repetitive region of the H. echinata Piwil promoter (Suppl. File S1; F- CAGATGATCCGCAGACAATAG, RAAATGTAATGAAAATTTTCGTAATTA) or the Widom '601' sequence (FCTGCAGAAGCTTGGTCCC, R-ACAGGATGTATATATCTG) were used for the $2080 \mathrm{bp}$ and $147 \mathrm{bp}$ fragments, respectively. PCR wells were pooled and ethanol precipitated by adding 0.1 volumes of $3 \mathrm{M}$ sodium acetate $\mathrm{pH} 5.2$ and 2.5 volumes of absolute ethanol then centrifuging at $11,000 \mathrm{G}$ at $4^{\circ} \mathrm{C}$ for $30 \mathrm{~min}$. The DNA pellet was

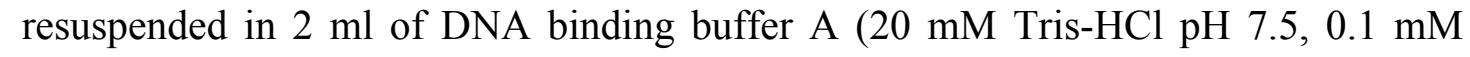
EDTA). Finally, DNA was purified by ion exchange chromatography as described above. 


\section{Nucleosome assembly by salt dialysis}

To assemble $250 \mathrm{pmol}$ of nucleosomes in a volume of $80 \mu \mathrm{l}$, purified octamer was mixed with the appropriate DNA in an equimolar ratio of nucleosome sites in a solution containing a final concentration of $20 \mathrm{mM}$ Tris- $\mathrm{HCl} \mathrm{pH} 7.5$ and $2 \mathrm{M} \mathrm{NaCl}$. The reaction mixture was transferred into a custom-made miniature dialysis block pre-equilibrated at $4^{\circ} \mathrm{C}$ with $8,000 \mathrm{MWCO}$ dialysis membranes. Samples were dialysed against solutions containing $10 \mathrm{mM}$ Tris-HCl pH 7.5 and $1 \mathrm{mM}$ EDTA with consecutively $1.4 \mathrm{M}, 1.2 \mathrm{M}, 0.8 \mathrm{M}$ and $0.6 \mathrm{M} \mathrm{NaCl}$ for at least $2 \mathrm{~h}$ each, and then finally against $10 \mathrm{mM}$ Tris- $\mathrm{HCl} \mathrm{pH} 7.5$ overnight. Nucleosomal arrays were visualised by mixed agarose-polyacrylamide native gel electrophoresis, and mononucleosomes were visualised by native PAGE.

\section{Mixed agarose-polyacrylamide native gel electrophoresis}

Gels comprising $1 \%$ agarose and $2 \%$ acrylamide with $1: 37.5$ bisacrylamide in $0.2 \mathrm{x}$ TBE were heated to dissolve agarose, where $1 \mathrm{x}$ TBE is $89 \mathrm{mM}$ Tris $\mathrm{pH} 7.5,89 \mathrm{mM}$ borate, $2 \mathrm{mM}$ EDTA. The solution was allowed to cool to $\sim 40^{\circ} \mathrm{C}$ before adding $0.1 \%$ TEMED and ammonium persulphate to $0.1 \%$ then pouring into pre-warmed $16 \mathrm{~cm} \mathrm{x}$ $20 \mathrm{~cm}$ glass plates with $1 \mathrm{~mm}$ spacers. Polymerised gels were pre-equilibrated for 145 min at $250 \mathrm{~V}$ in $0.2 \mathrm{x}$ TBE Buffer. Samples were mixed with 0.7 volumes of $10 \mathrm{mM}$ Tris- $\mathrm{HCl} \mathrm{pH} 7.5,6 \%$ sucrose. Electrophoresis was performed under the same conditions as pre-equilibration.

\section{Nucleosome array digestion by ApoI}

Nucleosome arrays were digested with ApoI restriction enzyme by mixing 15 pmol assembled nucleosomal array in $50 \mu$ volume with 1x NEB CutSmart buffer and 7.5 units of restriction enzyme at $37^{\circ} \mathrm{C} .10 \mu \mathrm{L}$ samples were withdrawn at each time point and mixed rapidly with $33 \%$ phenol and $33 \%$ chloroform to stop the reaction. The aqueous phase was separated by centrifugation then ethanol precipitated, and the DNA pellet was resuspended in 1x DNA loading dye. Digestion was visualised by native PAGE. 


\section{Native PAGE}

A 6\% acrylamide with 1:37.5 bisacrylamide native polyacrylamide gel was cast and left for $1 \mathrm{~h}$ at RT for complete polymerization. The gel was thermally equilibrated for $1 \mathrm{~h}$ at $4^{\circ} \mathrm{C}$ before being pre-run for $3 \mathrm{~h}$ at $250 \mathrm{~V}$ and $4^{\circ} \mathrm{C}$ in $0.2 \mathrm{x}$ TBE. 4 pmol of nucleosome or DNA in 5\% sucrose was loaded per lane in native PAGE and the gel was run for a further $3 \mathrm{~h}$ at $250 \mathrm{~V}$ at $4^{\circ} \mathrm{C}$ in $0.2 \mathrm{x}$ TBE.

\section{Imaging of native polyacrylamide gels}

Gels with unlabelled DNA were stained with $10 \mathrm{ng} \mathrm{ml}^{-1}$ ethidium bromide for $15 \mathrm{~min}$ then scanned using a Fuji FLA5100 or BioRad Pharos FX fluorescent imager with the appropriate laser and filter settings for $\mathrm{Cy} 3, \mathrm{Cy} 5$ or ethidium bromide.

\section{Thermal stability assay by fluorescent dye binding}

A $20 \mu \mathrm{L}$ reaction mix was prepared by mixing $36 \mathrm{pmol}$ of nucleosomes assembled on 147 bp Widom 601 DNA with freshly diluted 5x SyPro Orange with $0.1 \mathrm{mM} \mathrm{NaCl}$, 20 mM Tris-Cl, pH 7.5 in a 96 well MicroAmp Fast optical plate. Thermal unfolding analysed on StepOne Plus Thermal Cycler as described in (Taguchi et al., 2014).

\section{Cell counting and image analysis}

The Automated Counting feature of ImageJ/Fiji (https://fiji.sc/) was used for cell counting. For EdU positive nuclei counting, confocal Z-stacks were projected. Color images (RGB) were first converted into greyscale before proceeding through the following settings: 'Edit - Options - Conversations' to 'scale when converting.' Then images were convert to greyscale using 'Image - Type - 16-bit'. All structures to be counted were highlighted according to the following settings: 'Image - Adjust Threshold'. The sliders were then used to highlight structures and settings were applied to create a binary version of the image with pixel intensities black $=0$ and white $=255$. When particles overlapped the 'Process - Binary - Watershed' function was applied to separate then by adding a 1 pixel thick line. Following this, the 'Analyze - Analyze Particles' function was used. In order to avoid counting noise, particle size was adjusted excluding noise-derived particles. The outlines of cell nuclei were then appearing together with a summary table showing the number of cells counted. These results were then used for statistical analysis. Cell counting 
470

471

472

experiments are shown as data from 2-3 independent technical replicates, with 15-20 biological replicates in each group. Data was normalised and $p$ values were calculated using T-test in the SPSS software package.

\section{Analysis of nuclear areas}

Nuclei of intact male sexual polyps were stained by Hoechst and confocal images from early to late stages of spermatogenesis were taken, along with epithelial somatic cells from the surface of the body column with the same magnifications and settings. The perimeter of 100 nuclei in an optical section of each sample was defined manually and the pixels counted as a measurement of area.

\section{Analysis of native gel migration}

Individual lanes from immediately below the well to the smallest DNA band were cropped from TIFF images and read as pixelwise arrays then summed by row. Background was estimated from the region of $97 \%$ migration in image and subtracted, then the row-wise cumulative intensity was calculated and normalised to the maximum. The relative migration of the $50^{\text {th }}$ percentile for the cumulative signal was extracted as the Rf of median DNA band intensity versus ApoI digestion time. Time series data for histone octamers containing each histone variant (H2B.1 n=3, H2B.3 $\mathrm{n}=2$, H2B.6 $\mathrm{n}=1$ ) were fitted to a $\log$ normal function using curve_fit from the optimize module of SciPy. All data was plotted using seaborn with appropriate matplotlib parameters. 
bioRxiv preprint doi: https://doi.org/10.1101/2021.08.30.458175; this version posted August 30, 2021. The copyright holder for this preprint

(which was not certified by peer review) is the author/funder, who has granted bioRxiv a license to display the preprint in perpetuity. It is made available under aCC-BY-NC-ND 4.0 International license.

\section{$491 \quad$ Figures}

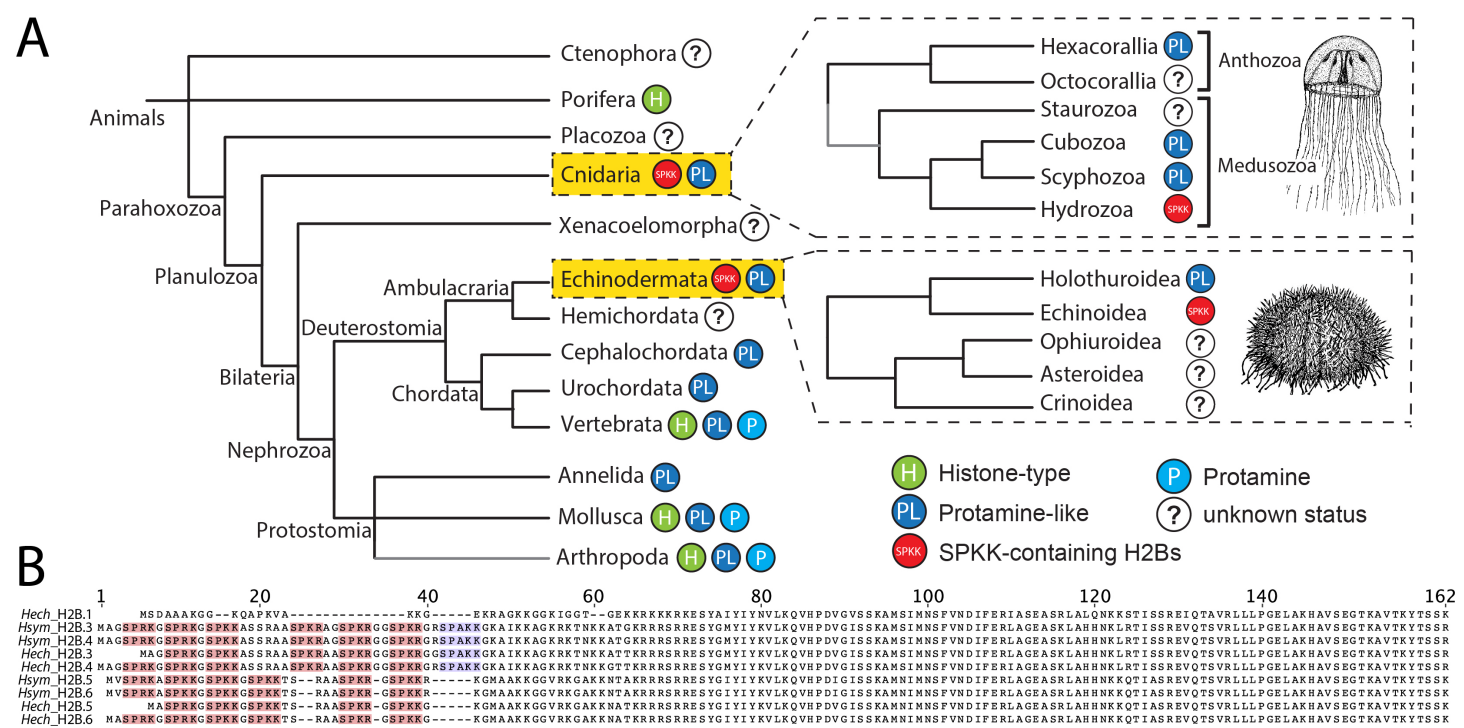

Figure 1: The evolution of N-terminal SPKK-related motif-containing H2B

494 histones. (A) Cladogram depicting the distribution of SNBPs in animals. (B) Protein

495 alignment showing $H$. echinata and $H$. symbiolongicarpus canonical and $496 \mathrm{SP}[\mathrm{K} / \mathrm{R}][\mathrm{K} / \mathrm{R}]$-containing H2Bs. 

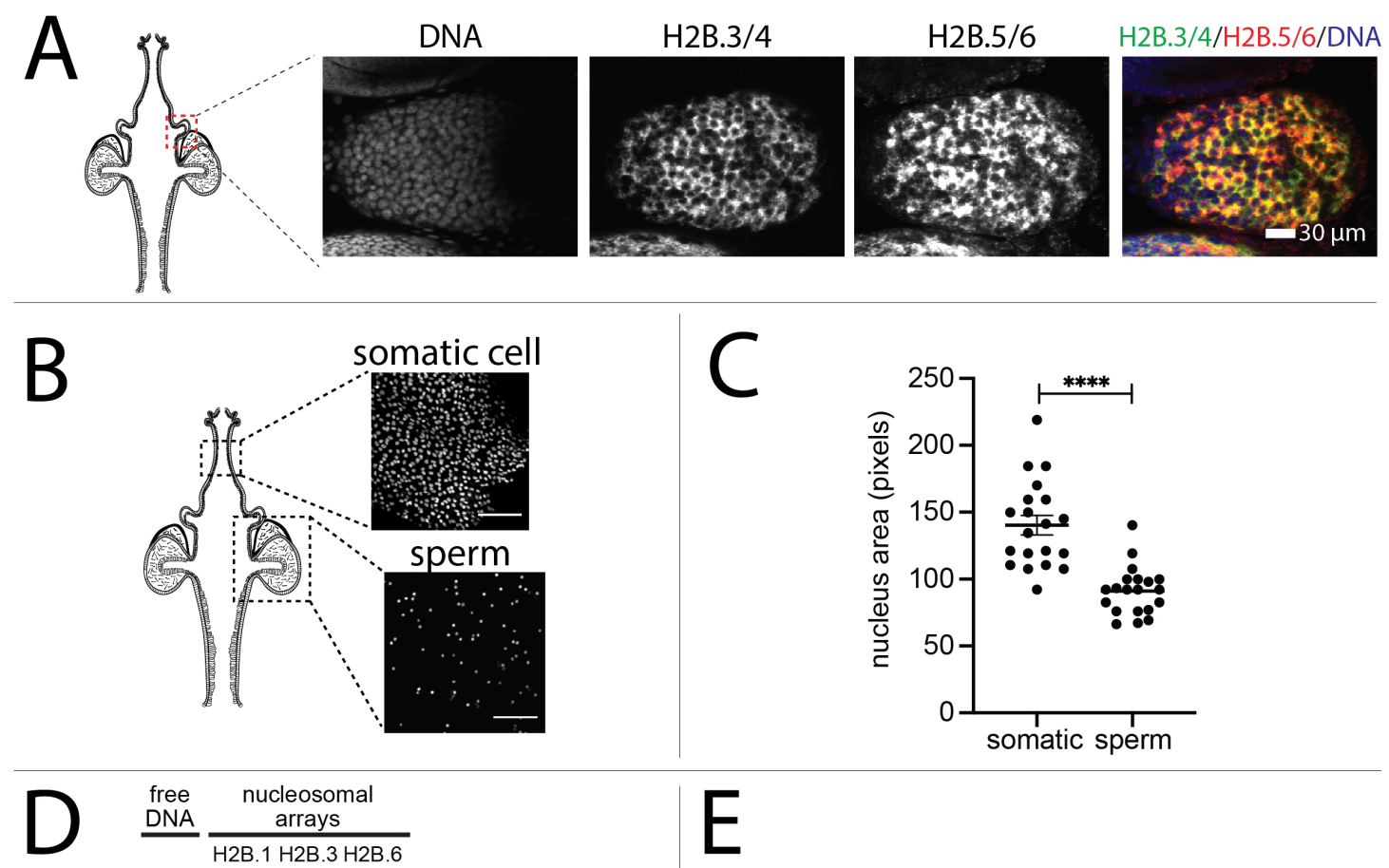

$E$
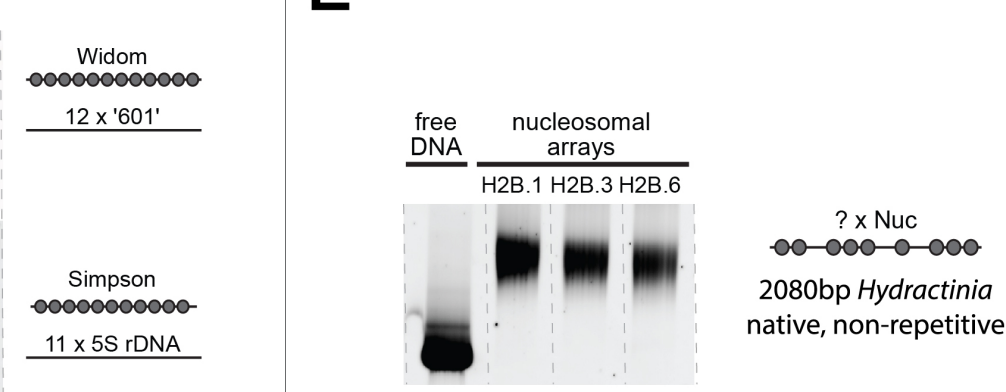

Figure 2: spH2Bs are co-expressed, replace H2B.1, but do not enhance chromatin compaction. (A) Expression of $H 2 B .3 / H 2 B .4$ and $H 2 B .5 / H 2 B .6$ in sperm progenitor cells visualised by RNA FISH.

(B) Hoechst staining of DNA in diploid somatic cells and mature haploid sperm cells for nuclear area measurements. Scale

502 bar $10 \mu \mathrm{m}$. (C) Distribution of nuclear areas for cell types in $B(n=20)$. (D) Native

503 PAGE of nucleosome arrays assembled on 12x 177 bp 601 repeat ('Widom') and 11x

504208 bp 5S rDNA repeat ('Simpson') using histone octamers containing either H2B.1, 505 H2B.3 or H2B.6. (E) Native PAGE of a 2080 bp non-repetitive Hydractinia DNA 506 using the same octamers as in (E). 
A
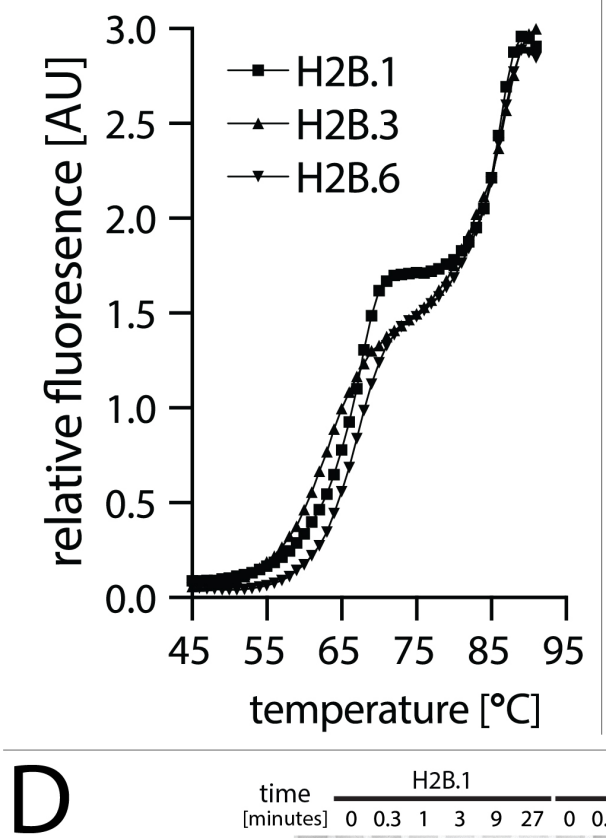

time
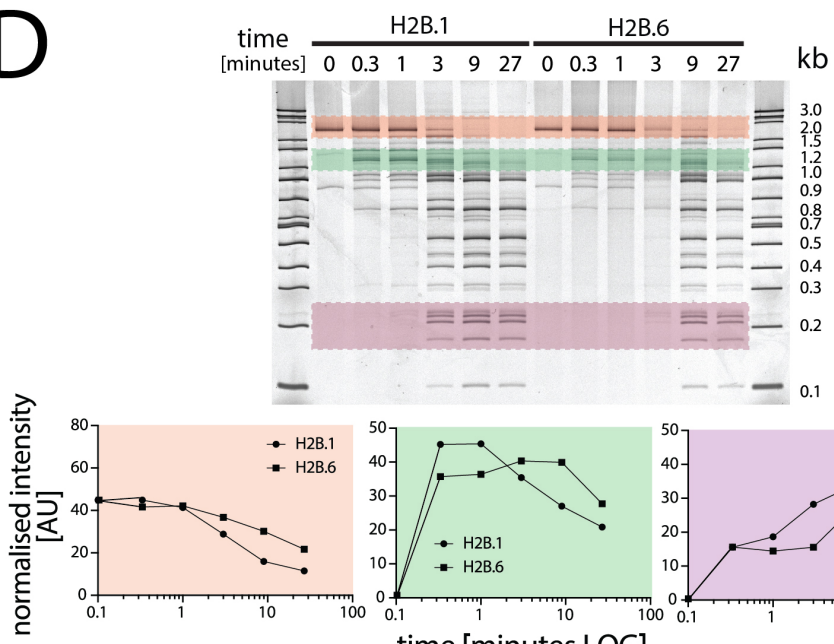

time [minutes LOG]
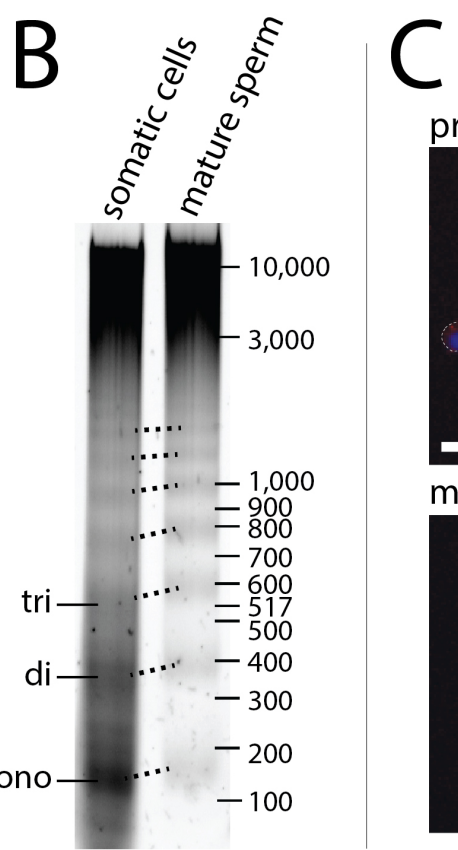

progenitors

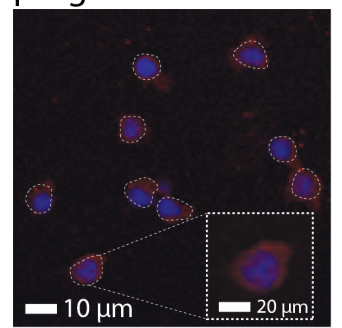

mature sperm
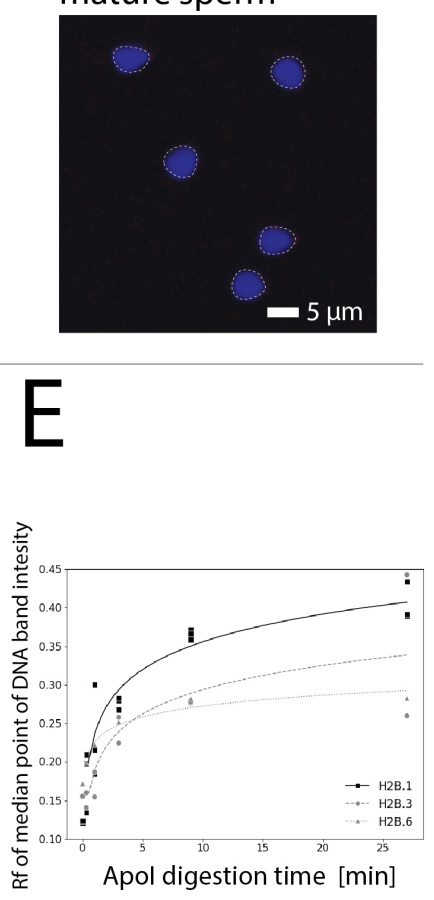

Figure 3: $\operatorname{spH2Bs}$ stabilise chromatin structure and restrict chromatin accessibility in vivo and in vitro. (A) Stability of mononucleosomes containing recombinant H2B.1, H2B.3, or H2B.6 measured by temperature dependence of

511 fluorescent dye binding in vitro. (B) Difference in nucleosomal repeat for somatic and 512 mature sperm cells after MNase digestion in vivo. (C) Mature sperm are inaccessible 513 to Tn5 integrase (red) comparing to progenitors. Nuclei were counterstained with 514 Hoechst (blue). (D) Native PAGE of equimolar recombinant H2B.1 or H2B.6containing nucleosome arrays on the 2080 bp non-repetitive Hydractinia Piwil 5'

516 non-coding region DNA digested by ApoI restriction enzyme. Band intensities for 3 517 representative DNA bands are shown (pink, red and purple). (E) Quantification of 518 native PAGE migration (Rf) for median point of DNA band intensity for ApoI 
bioRxiv preprint doi: https://doi.org/10.1101/2021.08.30.458175; this version posted August 30, 2021 . The copyright holder for this preprint

(which was not certified by peer review) is the author/funder, who has granted bioRxiv a license to display the preprint in perpetuity. It is made available under aCC-BY-NC-ND 4.0 International license.

519 digestion of H2B.1, H2B.3 or H2B.6-containing nucleosomal arrays from 4 replicate

520 native PAGE, showing reduced accessibility of H2B.6 nucleosomes in vitro.

521 

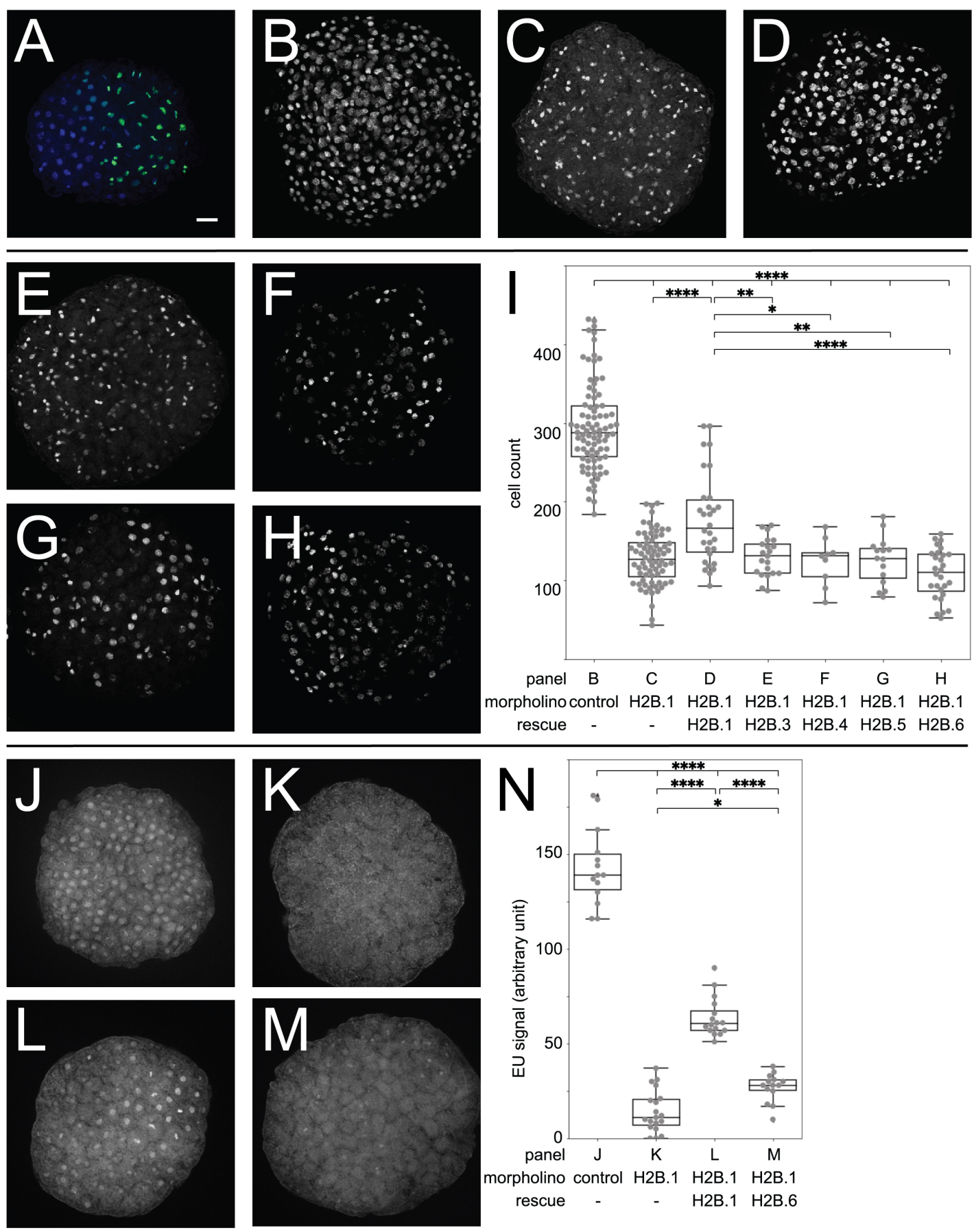

524 Figure 4: $\mathbf{s p H 2 B s}$ cause a transcription block and cell cycle arrest in the absence

525 of H2B.1. (A) GFP fluorescence 7 hours after injection of GFP control mRNA into 526 embryos. (B-H) Embryos stained with Hoechst after injection with (B) control 527 morpholino, (C) H2B.1 translation-blocking morpholino, (D) H2B.1-blocking 528 morpholino and morpholino-resistant H2B.1 mRNA, (E) H2B.1-blocking morpholino 529 and H2B.3 mRNA, (F) H2B.1-blocking morpholino and H2B.4 mRNA, (G) H2B.1- 
530 blocking morpholino and H2B.5 mRNA, (H) H2B.1-blocking morpholino an H2B.6

531 mRNA. (I) Distribution of cell counts in embryos from panels B-H. (J) EU 532 incorporation in embryo injected with control morpholino. (K) EU incorporation in 533 embryo injected with H2B.1-blocking morpholino. (L) EU incorporation in embryo 534 injected with H2B.1-blocking morpholino and morpholino-resistant H2B.1 mRNA. 535 (M) EU incorporation in embryo injected with H2B.1-blocking morpholino and 536 H2B.6 mRNA. N. Quantification of EU signals in embryos from panels J-M. The 537 scale bars equal $20 \mu \mathrm{m}$.

538 


\section{Declarations}

\section{Ethics approval and consent to participate}

542 Not applicable.

\section{Consent for publication}

544 All authors approve the manuscript.

$545 \quad$ Availability of data and material

546 Scripts for generating data plots are available at https://github.com/af-lab/hydractinia.

547 Materials including bacterial vector for Hydractinia histones can be obtain from the

548 laboratory of UF upon request.

\section{Competing interests}

550 The authors declare no competing interests.

$551 \quad$ Funding

552 This work was funded by Science Foundation Ireland (13/SIRG/2125 to SGG, 11-PI-1020 to

553 UF), Wellcome Trust (grant no. 210722/Z/18/Z, co-funded by the SFI-HRB-Wellcome

554 Biomedical Research Partnership to UF), and by the European Commission Marie Curie

555 Actions (PIIFGA-2013-623748 to SGG). F was a Hardiman Scholar and also supported by

556 a Thomas Crawford Hayes Research Grant.

\section{Authors' contributions}

558 Conceptualization: SGG, UF and AF; Methodology: AT, MGB, TQD, SGG; Investigation:

559 AT, MGB, JCV, IP, TQD, EA, F, SGG; Software: AF; Data Curating: AF, SGG; Writing:

560 AT, SGG, AF, UF; Funding Acquisition, SGG, UF; Supervision, SGG, AF, UF.

\section{Acknowledgements}

562 We thank members of our labs for lively discussions. Confocal images were taken at the 563 Centre for Microscopy and Imaging Core Facility at NUI Galway. 
Ausio, J. (1999). Histone H1 and Evolution of Sperm Nuclear Basic Proteins. J Biol Chem 274.

Ausio, J., Veghel, M. L., Gomez, R. and Barreda, D. (1997). The sperm nuclear basic proteins (SNBPs) of the sponge Neofibularia nolitangere: implications for the molecular evolution of SNBPs. J Mol Evol 45.

Buenrostro, J. D., Giresi, P. G., Zaba, L. C., Chang, H. Y. and Greenleaf, W. J. (2013). Transposition of native chromatin for fast and sensitive epigenomic profiling of open chromatin, DNA-binding proteins and nucleosome position. Nat Meth 10, 1213-1218.

Busslinger, M. and Barberis, A. (1985). Synthesis of sperm and late histone cDNAs of the sea urchin with a primer complementary to the conserved 3 ' terminal palindrome: evidence for tissue-specific and more general histone gene variants. Proc Natl Acad Sci U S A 82, 5676-5680.

Champroux, A., Torres-Carreira, J., Gharagozloo, P., Drevet, J. R. and Kocer, A. (2016). Mammalian sperm nuclear organization: resiliencies and vulnerabilities. Basic Clin Androl 26, 17.

Churchill, M. E. and Suzuki, M. (1989). 'SPKK' motifs prefer to bind to DNA at A/T-rich sites. The EMBO journal 8, 4189-4195.

Dorigo, B., Schalch, T., Bystricky, K. and Richmond, T. J. (2003). Chromatin fiber folding: requirement for the histone H4 N-terminal tail. J Mol Biol 327, 85-96.

Frank, U., Nicotra, M. L. and Schnitzler, C. E. (2020). The colonial cnidarian Hydractinia. Evodevo 11.

Fuentes-Mascorro, G., Serrano, H. and Rosado, A. (2000). Sperm chromatin. Arch Androl 45, 215-225.

Green, G. R. and Poccia, D. L. (1985). Phosphorylation of sea urchin sperm H1 and $\mathrm{H} 2 \mathrm{~B}$ histones precedes chromatin decondensation and $\mathrm{H} 1$ exchange during pronuclear formation. Developmental Biology 108, 235-245.

---- (1988). Interaction of sperm histone variants and linker DNA during spermiogenesis in the sea urchin. Biochemistry 27, 619-625.

Hill, C. S. and Thomas, J. O. (1990). Core histone-DNA interactions in sea urchin sperm chromatin. The N-terminal tail of H2B interacts with linker DNA. Eur J Biochem 187, 145-153.

Khadake, J. R. and Rao, M. R. (1997). Condensation of DNA and chromatin by an SPKK-containing octapeptide repeat motif present in the C-terminus of histone H1. Biochemistry 36, 1041-1051.

Levitan, D. R. and Petersen, C. (1995). Sperm limitation in the sea. Trends Ecol Evol 10, 228-231.

Logie, C. and Peterson, C. L. (1997). Catalytic activity of the yeast SWI/SNF complex on reconstituted nucleosome arrays. Embo j 16, 6772-6782.

Lowary, P. T. and Widom, J. (1998). New DNA sequence rules for high affinity binding to histone octamer and sequence-directed nucleosome positioning. $J$ Mol Biol 276, 19-42.

Malik, H. S., Vermaak, D. and Henikoff, S. (2002). Recurrent evolution of DNAbinding motifs in the $<\mathrm{em}>$ Drosophila $</ \mathrm{em}>$ centromeric histone. Proceedings of the National Academy of Sciences 99, 1449-1454.

Marzluff, W. F., Sakallah, S. and Kelkar, H. (2006). The sea urchin histone gene complement. Dev Biol 300, 308-320. 
Pérez-Montero, S., Carbonell, A. and Azorín, F. (2016). Germline-specific H1 variants: the "sexy" linker histones. Chromosoma 125.

Picelli, S., Bjorklund, A. K., Reinius, B., Sagasser, S., Winberg, G. and Sandberg, R. (2014). Tn5 transposase and tagmentation procedures for massively scaled sequencing projects. Genome Res 24, 2033-2040.

Poccia, D. L. and Green, G. R. (1992). Packaging and unpackaging the sea urchin sperm genome. Trends Biochem Sci 17.

Poccia, D. L., Simpson, M. V. and Green, G. R. (1987). Transitions in histone variants during sea urchin spermatogenesis. Dev Biol 121, 445-453.

Rocchini, C., Marx, R. M. and Carosfeld, J. S. (1996). Replacement of nucleosomal histones by histone H1-like proteins during spermiogenesis in Cnidaria: evolutionary implications. J Mol Evol 42.

Sassone-Corsi, P. (2002). Unique chromatin remodeling and transcriptional regulation in spermatogenesis. Science 296, 2176-2178.

Simpson, R. T., Thoma, F. and Brubaker, J. M. (1985). Chromatin reconstituted from tandemly repeated cloned DNA fragments and core histones: a model system for study of higher order structure. Cell 42, 799-808.

Suzuki, M. (1989). SPKK, a new nucleic acid-binding unit of protein found in histone. Embo j 8, 797-804.

Suzuki, M., Gerstein, M. and Johnson, T. (1993). An NMR study on the DNAbinding SPKK motif and a model for its interaction with DNA. Protein Eng 6, 565-574.

Suzuki, M., Sugiura, M. and Ebashi, S. (1990). Sea urchin protease specific to the SPKK motif in histone. J Biochem 108, 347-355.

Taguchi, H., Horikoshi, N., Arimura, Y. and Kurumizaka, H. (2014). A method for evaluating nucleosome stability with a protein-binding fluorescent dye. Methods 70, 119-126.

Török, A. and Gornik, S. G. (2018). Sperm Nuclear Basic Proteins of Marine Invertebrates. Results Probl Cell Differ 65, 15-32.

Török, A., Schiffer, P. H., Schnitzler, C. E., Ford, K., Mullikin, J. C., Baxevanis, A. D., Bacic, A., Frank, U. and Gornik, S. G. (2016). The cnidarian Hydractinia echinata employs canonical and highly adapted histones to pack its DNA. Epigenetics Chromatin 9, 36.

Ward, W. S. and Coffey, D. S. (1991). DNA packaging and organization in mammalian spermatozoa: comparison with somatic cells. Biology of reproduction 44, 569-574. 